\title{
Prediction of Cancer Incidence and Mortality in Korea, 2017
}

Kyu-Won Jung, MS',2
Young-Joo Won, $\mathrm{PhD}^{1,2,3}$
Chang-Mo Oh, MD, $\mathrm{PhD}^{1,2}$
Hyun-Joo Kong, MS
Duk Hyoung Lee, MD, $\mathrm{PhD}^{2,3}$
Kang Hyun Lee, MD, $\mathrm{PhD}^{1}$

${ }^{1}$ The Korea Central Cancer Registry, National Cancer Center, Goyang, ${ }^{2}$ National Cancer Control Institute, National Cancer Center, Goyang, ${ }^{3}$ Department of Cancer Control and Population Health, National Cancer Center Graduate School of Cancer Science and Policy, Goyang, Korea

\begin{abstract}
Purpose
This study aimed to report on cancer incidence and mortality for the year 2017 in Korea in order to estimate the nation's current cancer burden.

\section{Materials and Methods}

Cancer incidence data from 1999 to 2014 were obtained from the Korea National Cancer Incidence Database, and cancer mortality data from 1993 to 2015 were acquired from Statistics Korea. Cancer incidence and mortality were projected by fitting a linear regression model to observe age-specific cancer rates against observed years, and then multiplying the projected age-specific rates by the age-specific population. The Joinpoint regression model was used to determine at which year the linear trend changed significantly; we only used data of the latest trend.
\end{abstract}

\section{Results}

A total of 221,143 new cancer cases and 80,268 cancer deaths are expected to occur in Korea in 2017. The most common cancer sites are the colorectum, stomach, lung, thyroid, and breast. These five cancers represent half of the overall burden of cancer in Korea. For mortality, the most common sites are the lung, liver, colorectal, stomach, and pancreas.

\section{Conclusion}

The incidence rate of all cancers in Korea appears to have decreased mainly because of a decrease in thyroid cancer. These up-to-date estimates of the cancer burden in Korea could be an important resource for planning and evaluation of cancer-control programs.

\section{Key words}

Incidence, Mortality, Neoplasms, Forecasting, Korea, 2017

\section{Introduction}

As the leading cause of death in Korea [1], cancer has been the country's major public health concern since 1983. Over 210,000 patients were newly diagnosed with cancer in Korea, and one in four deaths is due to cancer [2]. Although the cancer registration system in Korea is highly efficient and can provide nationwide cancer statistics within a relatively brief period, a lag time of at least 2 years is required to collect and analyze the data of a specific year. For planning and implementation of comprehensive cancer control programs, it is important to assess the number of new cases and deaths that are expected to occur during the current year. In this study, we report the projected cancer incidence and mortality for the year 2017 based on the data from the 1990s through 2015. 


\section{Materials and Methods}

The Korean Ministry of Health and Welfare initiated a nationwide, hospital-based cancer registry, the Korea Central Cancer Registry (KCCR), in 1980. The history, objectives, and activities of the KCCR have been documented in detail elsewhere [3]. Incidence data from 1999 to 2014 were obtained from the Korea National Cancer Incidence Database (KNCI DB). Cancer cases were classified according to the International Classification of Diseases for Oncology, third edition [4], and converted according to the International Classification of Diseases, 10th edition (ICD-10) [5]. Mortality data from 1993 to 2015 were acquired from Statistics Korea [1]. The cause of death was coded and classified according to ICD-10 [5].

The cancer sites included in this study were (1) all cancers combined and (2) the 24 common cancers as follows: lip, oral cavity, and pharynx (C00-C14), esophagus (C15), stomach
(C16), colon and rectum (C18-C20), liver and intrahepatic bile duct (liver) (C22), gallbladder and other parts of the biliary tract (gallbladder) (C23-C24), pancreas (C25), larynx (C32), lung and bronchus (lung) (C33-C34), breast (C50), cervix uteri (C53), corpus uteri (C54), ovary (C56), prostate (C61), testis (C62), kidney (C64), bladder (C67), brain and central nervous system (C70-C72), thyroid (C73), Hodgkin lymphoma (C81), non-Hodgkin lymphoma (C82-C85, C96), multiple myeloma (C90), leukemia (C91-C95), and others.

Population data from 1993 to 2017 were obtained from the resident registration population, as reported by Statistics Korea [1]. Data on the mid-year population, as of July 1 of the respective year, were analyzed. For the year 2017, however, we used population data as of December 31, 2016, because mid-2016 resident registration population was not yet available at the time of analysis.

Linear regression models [6] were used to assess time trends and projections. Firstly, we first performed a Joinpoint regression analysis of the data available to detect the year

Table 1. Estimated new cancer cases and deaths by sex during 2017 in Korea

\begin{tabular}{|c|c|c|c|c|c|c|}
\hline \multirow{2}{*}{ Site } & \multicolumn{3}{|c|}{ Estimated new cases } & \multicolumn{3}{|c|}{ Estimated deaths } \\
\hline & Both sexes & Male & Female & Both sexes & Male & Female \\
\hline All sites & 221,143 & 117,990 & 103,153 & 80,268 & 49,446 & 30,822 \\
\hline Lip, oral cavity, and pharynx & 3,477 & 2,526 & 951 & 1,146 & 841 & 305 \\
\hline Esophagus & 2,457 & 2,246 & 211 & 1,275 & 1,196 & 79 \\
\hline Stomach & 26,350 & 17,548 & 8,802 & 7,480 & 4,841 & 2,639 \\
\hline Colon and rectum & 27,837 & 16,096 & 11,741 & 9,224 & 5,477 & 3,747 \\
\hline Liver & 15,593 & 11,881 & 3,712 & 11,057 & 8,128 & 2,929 \\
\hline Gallbladder ${ }^{\text {a) }}$ & 6,343 & 3,297 & 3,046 & 4,349 & 2,121 & 2,228 \\
\hline Pancreas & 7,071 & 3,765 & 3,306 & 6,029 & 3,144 & 2,885 \\
\hline Larynx & 1,066 & 1,035 & 31 & 324 & 322 & 2 \\
\hline Lung & 26,093 & 18,371 & 7,722 & 18,713 & 13,567 & 5,146 \\
\hline Breast & 21,623 & 79 & 21,544 & 2,609 & 18 & 2,591 \\
\hline Cervix uteri & 2,962 & - & 2,962 & 784 & - & 784 \\
\hline Corpus uteri & 2,578 & - & 2,578 & 338 & - & 338 \\
\hline Ovary & 2,618 & - & 2,618 & 1,168 & - & 1,168 \\
\hline Prostate & 11,646 & 11,646 & - & 2,067 & 2,067 & - \\
\hline Testis & 279 & 279 & - & 18 & 18 & - \\
\hline Kidney & 5,141 & 3,558 & 1,583 & 1,131 & 787 & 344 \\
\hline Bladder & 4,190 & 3,353 & 837 & 1,495 & 1,124 & 371 \\
\hline Brain and CNS & 1,783 & 936 & 847 & 1,285 & 650 & 635 \\
\hline Thyroid & 23,862 & 6,275 & 17,587 & 360 & 104 & 256 \\
\hline Hodgkin lymphoma & 319 & 202 & 117 & 66 & 38 & 28 \\
\hline Non-Hodgkin lymphoma & 5,685 & 3,270 & 2,415 & 1,803 & 1,038 & 765 \\
\hline Multiple myeloma & 1,726 & 931 & 795 & 1,018 & 555 & 463 \\
\hline Leukemia & 3,401 & 1,939 & 1,462 & 1,810 & 1,026 & 784 \\
\hline Other and ill defined & 17,043 & 8,757 & 8,286 & 4,719 & 2,384 & 2,335 \\
\hline
\end{tabular}

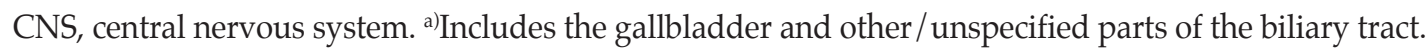




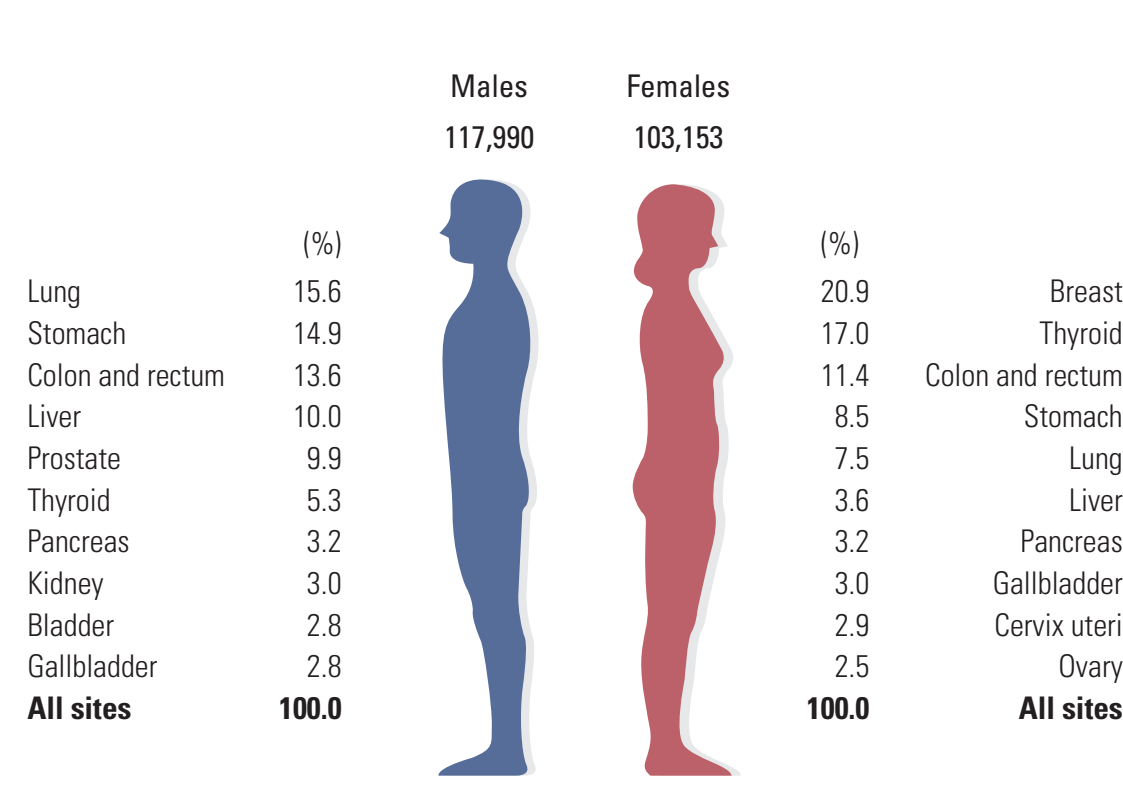

B

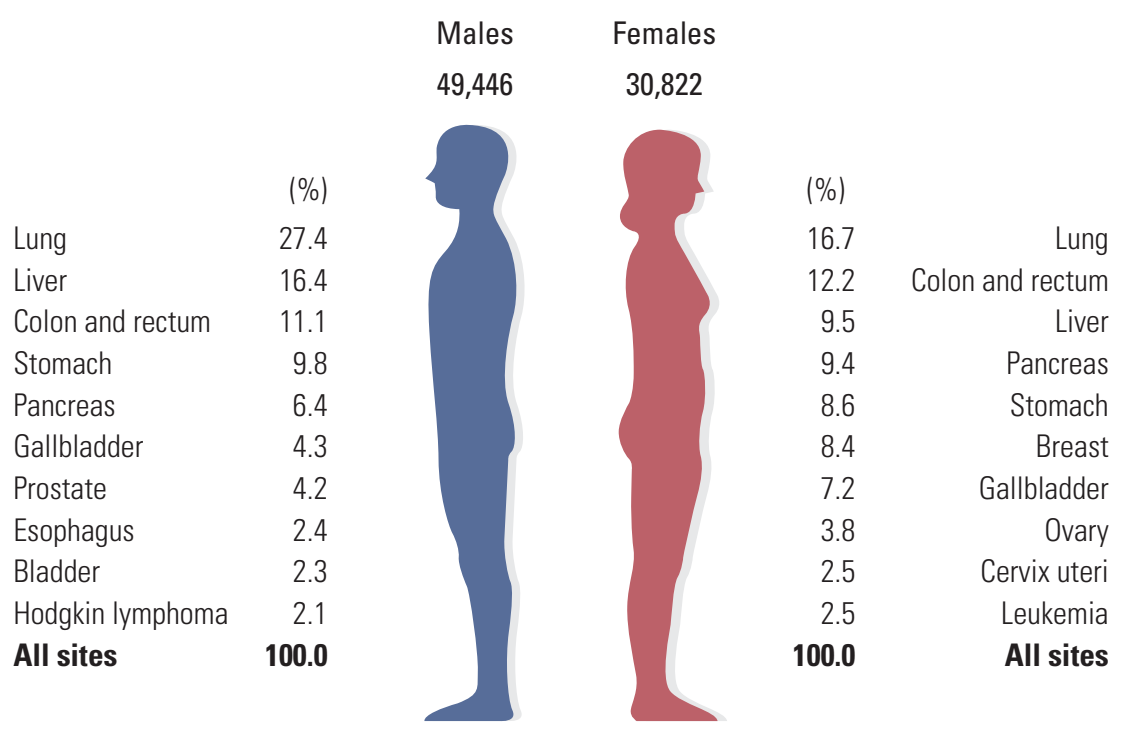

Fig. 1. The 10 leading types of estimated new cancer cases and deaths by sex in 2017. (A) Estimated new cases. (B) Estimated deaths.

when significant changes occurred in cancer trends according to sex and cancer site. A Joinpoint regression describes changes in data trends by connecting several different line segments on a log scale at "joinpoints." This analysis was performed using the Joinpoint software (ver. 4.0.1, http:// surveillance.cancer.gov/joinpoint) from the Surveillance
Research Program of the US National Cancer Institute [7]. We chose the number of joinpoint in order to have least four data points between consecutive joinpoints. Secondly, to predict age-specific cancer rates, a linear regression model was fitted to age-specific rates by 5-year age groups against observed years based on observed cancer incidence data of 
Table 2. Estimated crude and age-standardized cancer incidences by sex during 2017 in Korea

\begin{tabular}{|c|c|c|c|c|c|c|}
\hline \multirow[t]{2}{*}{ Site } & \multicolumn{3}{|c|}{$\begin{array}{c}\text { Crude incidence rates } \\
\text { per } 100,000\end{array}$} & \multicolumn{3}{|c|}{$\begin{array}{l}\text { Age-standardized incidence rates } \\
\text { per } 100,000^{\text {a) }}\end{array}$} \\
\hline & Both sexes & Male & Female & Both sexes & Male & Female \\
\hline All sites & 427.8 & 456.8 & 398.8 & 251.3 & 282.6 & 234.6 \\
\hline Lip, oral cavity, and pharynx & 6.7 & 9.8 & 3.7 & 4.1 & 6.1 & 2.2 \\
\hline Esophagus & 4.8 & 8.7 & 0.8 & 2.5 & 5.1 & 0.4 \\
\hline Stomach & 51.0 & 67.9 & 34.0 & 28.3 & 41.1 & 17.4 \\
\hline Colon and rectum & 53.9 & 62.3 & 45.4 & 29.3 & 37.6 & 22.4 \\
\hline Liver & 30.2 & 46.0 & 14.4 & 16.6 & 27.7 & 6.7 \\
\hline Gallbladder ${ }^{b)}$ & 12.3 & 12.8 & 11.8 & 6.1 & 7.5 & 5.0 \\
\hline Pancreas & 13.7 & 14.6 & 12.8 & 7.1 & 8.7 & 5.8 \\
\hline Larynx & 2.1 & 4.0 & 0.1 & 1.1 & 2.4 & 0.1 \\
\hline Lung & 50.5 & 71.1 & 29.9 & 26.2 & 41.8 & 14.4 \\
\hline Breast & 41.8 & 0.3 & 83.3 & 26.9 & 0.2 & 53.5 \\
\hline Cervix uteri & 5.7 & - & 11.5 & 3.7 & - & 7.2 \\
\hline Corpus uteri & 5.0 & - & 10.0 & 3.2 & - & 6.3 \\
\hline Ovary & 5.1 & - & 10.1 & 3.3 & - & 6.6 \\
\hline Prostate & 22.5 & 45.1 & - & 12.0 & 26.7 & - \\
\hline Testis & 0.5 & 1.1 & - & 0.6 & 1.1 & - \\
\hline Kidney & 9.9 & 13.8 & 6.1 & 5.9 & 8.7 & 3.4 \\
\hline Bladder & 8.1 & 13.0 & 3.2 & 4.1 & 7.7 & 1.3 \\
\hline Brain and CNS & 3.5 & 3.6 & 3.3 & 2.5 & 2.7 & 2.3 \\
\hline Thyroid & 46.2 & 24.3 & 68.0 & 33.8 & 17.4 & 50.8 \\
\hline Hodgkin lymphoma & 0.6 & 0.8 & 0.5 & 0.5 & 0.7 & 0.4 \\
\hline Non-Hodgkin lymphoma & 11.0 & 12.7 & 9.3 & 7.2 & 8.8 & 5.8 \\
\hline Multiple myeloma & 3.3 & 3.6 & 3.1 & 1.8 & 2.2 & 1.5 \\
\hline Leukemia & 6.6 & 7.5 & 5.7 & 5.3 & 6.3 & 4.4 \\
\hline Other and ill defined & 33.0 & 33.9 & 32.0 & 19.3 & 22.3 & 16.7 \\
\hline
\end{tabular}

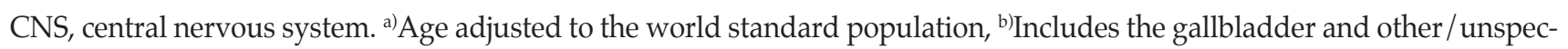
ified parts of the biliary tract.

the latest trend. Finally, we multiply the projected age-specific rates by the age-specific population to obtain the projected cancer cases and deaths for the year 2017.

We summarized the results using crude rates (CRs) and age-standardized rates (ASRs) of cancer incidence and mortality. ASRs were standardized using the world standard population [8] and expressed per 100,000 persons.

\section{Results}

\section{Incidence}

A total of 221,143 new cancer cases are anticipated in 2017 (Table 1, Fig. 1), with more male $(n=117,990)$ than female $(n=103,153)$ cancer patients expected in the coming year.

The projected CRs per 100,000 of all sites combined in 2017 are projected to be 456.8 and 398.8 in men and women, respectively (Table 2). The projected ASRs per 100,000 of all sites combined are 282.6 and 234.6, respectively. In men, the five leading primary sites of cancer are expected to be the lung (CR, 71.1; ASR, 41.8), stomach (CR, 67.9; ASR, 41.1), colon and rectum (CR, 62.3; ASR, 37.6), liver (CR, 46.0; ASR, 27.7), and prostate (CR, 45.1; ASR, 26.7), accounting for $64.0 \%$ of all new cancers in 2017.

In women, the five leading primary sites are expected to be the breast (CR, 83.3; ASR, 53.5), thyroid (CR, 68.0; ASR, 50.8), colon and rectum (CR, 45.4; ASR, 22.4), stomach (CR, 34.0; ASR, 17.4), and lung (CR, 29.9; ASR, 14.4), accounting for $65.3 \%$ of all new cancers (Fig. 1).

The predicted five most common cancer sites for 2017 by sex and age group are shown in Table 3. Leukemia and thy- 
Table 3. Estimated cancer incidence by age group and sex during 2017 in Korea

\begin{tabular}{|c|c|c|c|c|}
\hline \multirow{2}{*}{ Rank } & \multicolumn{4}{|c|}{ Age group (yr) } \\
\hline & $0-14$ & $15-34$ & $35-64$ & $\geq 65$ \\
\hline \multicolumn{5}{|l|}{ Male } \\
\hline 1 & Leukemia (5.0) & Thyroid (16.3) & Stomach $(68.7)$ & Lung (437.3) \\
\hline 2 & Non-Hodgkin lymphoma (2.6) & Non-Hodgkin lymphoma (3.4) & Colon and rectum (58.1) & Stomach (306.2) \\
\hline 3 & Brain and CNS (1.4) & Leukemia (3.4) & Liver (52.3) & Colon and rectum (295.1) \\
\hline 4 & Thyroid (0.4) & Colon and rectum (3.2) & Lung (43.8) & Prostate (293.3) \\
\hline 5 & Liver (0.3) & Testis (2.3) & Thyroid (36.1) & Liver (181.5) \\
\hline \multicolumn{5}{|c|}{ Female } \\
\hline 1 & Leukemia (4.1) & Thyroid (50.4) & Breast (144.4) & Colon and rectum (173.4) \\
\hline 2 & Non-Hodgkin lymphoma (2.2) & Breast (11.3) & Thyroid (103.5) & Stomach (124.5) \\
\hline 3 & Brain and CNS (1.7) & Cervix uteri (5.4) & Colon and rectum (38.1) & Lung (120.2) \\
\hline 4 & Ovary $(0.8)$ & Ovary (3.2) & Stomach (29.9) & Breast (83.8) \\
\hline 5 & Thyroid (0.5) & Stomach (2.5) & Lung (23.2) & Liver (64.5) \\
\hline
\end{tabular}

CNS, central nervous system.

A

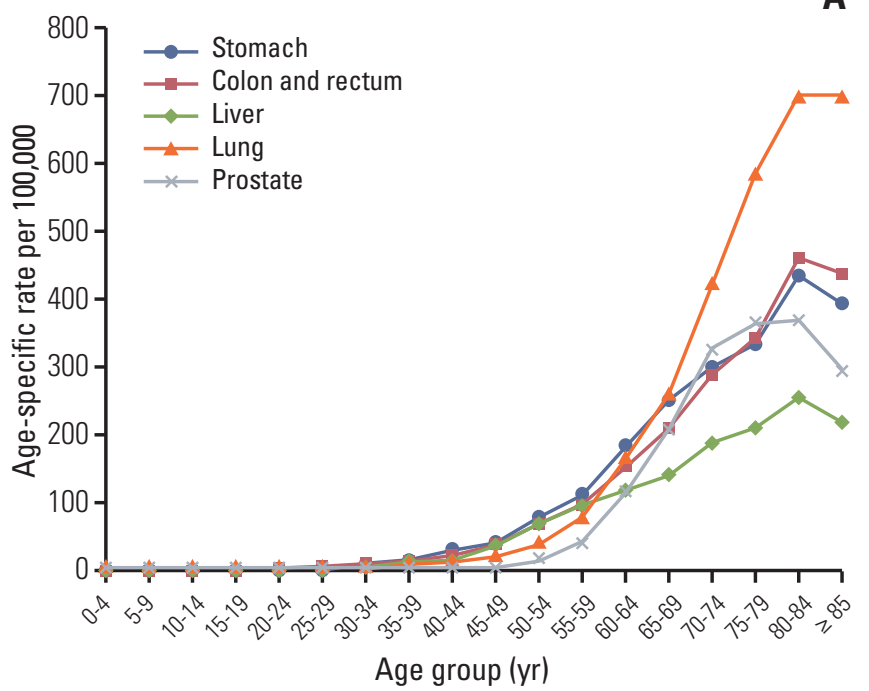

Fig. 2. Projected age-specific incidences of major cancers during 2017 in Korea. (A) Male. (B) Female.

roid cancer are expected to be the most common forms of cancer in both sexes for the 0-14 and 15-34 age groups. Stomach cancer is predicted to be the most prevalent in men aged 35-64 years, while lung cancer is expected to be more frequent in men aged 65 and over. Breast cancer is predicted to be the most common cancer in women 35-64 years of age, whereas colorectal cancer is expected to be the most prevalent in women aged 65 and over. These projections indicate that the incidences of stomach, lung, liver, colorectal, and prostate cancers will increase gradually with age for men

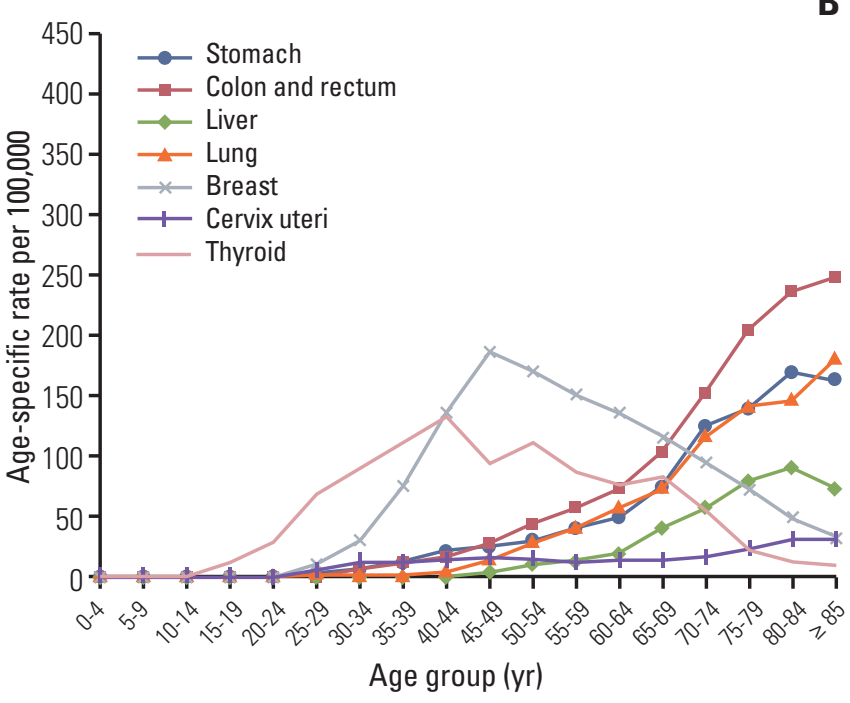

(Fig. 2). In women, the age-specific incidence rates of stomach, colorectal, liver, lung, and cervical cancers denote a rising trend in these cancers with age; however, the incidence of breast and thyroid cancer in women is expected to level off after the late 40 s and early 50 s, respectively.

\section{Mortality}

It is estimated that 80,268 cancer deaths will occur in Korea during 2017 (Table 1, Fig. 1). The projected CRs per 100,000 
Table 4. Estimated crude and age-standardized cancer mortality rates by sex during 2017 in Korea

\begin{tabular}{|c|c|c|c|c|c|c|}
\hline \multirow[t]{2}{*}{ Site } & \multicolumn{3}{|c|}{$\begin{array}{l}\text { Crude mortality rates } \\
\text { per } 100,000\end{array}$} & \multicolumn{3}{|c|}{$\begin{array}{c}\text { Age-standardized mortality rates } \\
\text { per } 100,000^{\mathrm{a})}\end{array}$} \\
\hline & Both sexes & Male & Female & Both sexes & Male & Female \\
\hline All sites & 155.3 & 191.5 & 119.2 & 77.5 & 112.7 & 52.2 \\
\hline Lip, oral cavity, and pharynx & 2.2 & 3.3 & 1.2 & 1.2 & 1.9 & 0.5 \\
\hline Esophagus & 2.5 & 4.6 & 0.3 & 1.2 & 2.7 & 0.1 \\
\hline Stomach & 14.5 & 18.7 & 10.2 & 7.0 & 11.0 & 4.1 \\
\hline Colon and rectum & 17.8 & 21.2 & 14.5 & 8.6 & 12.5 & 5.7 \\
\hline Liver & 21.4 & 31.5 & 11.3 & 11.2 & 18.7 & 4.8 \\
\hline Gallbladder ${ }^{\text {b) }}$ & 8.4 & 8.2 & 8.6 & 3.8 & 4.7 & 3.2 \\
\hline Pancreas & 11.7 & 12.2 & 11.2 & 5.8 & 7.2 & 4.6 \\
\hline Larynx & 0.6 & 1.3 & 0.0 & 0.3 & 0.7 & 0.0 \\
\hline Lung & 36.2 & 52.5 & 19.9 & 17.3 & 30.1 & 8.1 \\
\hline Breast & 5.1 & 0.1 & 10.0 & 3.0 & 0.0 & 5.8 \\
\hline Cervix uteri & 1.5 & - & 3.0 & 0.8 & - & 1.5 \\
\hline Corpus uteri & 0.7 & - & 1.3 & 0.4 & - & 0.7 \\
\hline Ovary & 2.3 & - & 4.5 & 1.2 & - & 2.4 \\
\hline Prostate & 4.0 & 8.0 & - & 1.7 & 4.7 & - \\
\hline Testis & 0.0 & 0.1 & - & 0.0 & 0.1 & - \\
\hline Kidney & 2.2 & 3.1 & 1.3 & 1.1 & 1.8 & 0.6 \\
\hline Bladder & 2.9 & 4.4 & 1.4 & 1.3 & 2.6 & 0.5 \\
\hline Brain and CNS & 2.5 & 2.5 & 2.5 & 1.6 & 1.8 & 1.5 \\
\hline Thyroid & 0.7 & 0.4 & 1.0 & 0.3 & 0.2 & 0.4 \\
\hline Hodgkin lymphoma & 0.1 & 0.2 & 0.1 & 0.1 & 0.1 & 0.1 \\
\hline Non-Hodgkin lymphoma & 3.5 & 4.0 & 3.0 & 1.8 & 2.4 & 1.3 \\
\hline Multiple myeloma & 2.0 & 2.2 & 1.8 & 1.0 & 1.3 & 0.8 \\
\hline Leukemia & 3.5 & 4.0 & 3.0 & 2.1 & 2.7 & 1.6 \\
\hline Other and ill defined & 9.1 & 9.2 & 9.0 & 4.7 & 5.7 & 4.0 \\
\hline
\end{tabular}

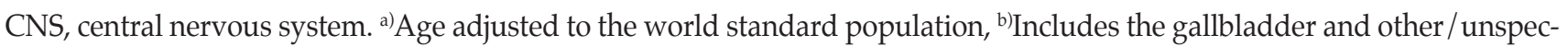
ified parts of the biliary tract.

A

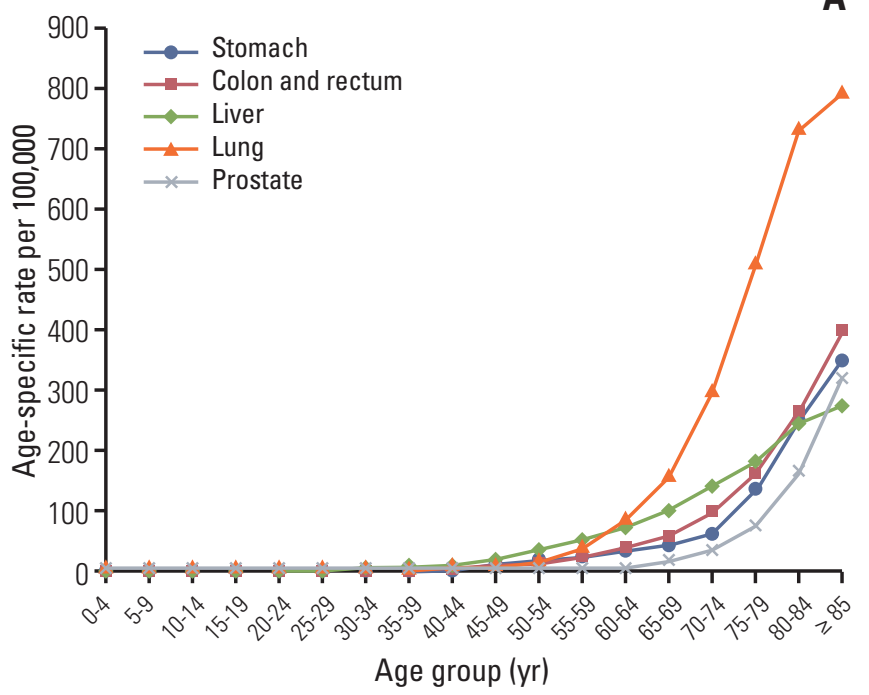

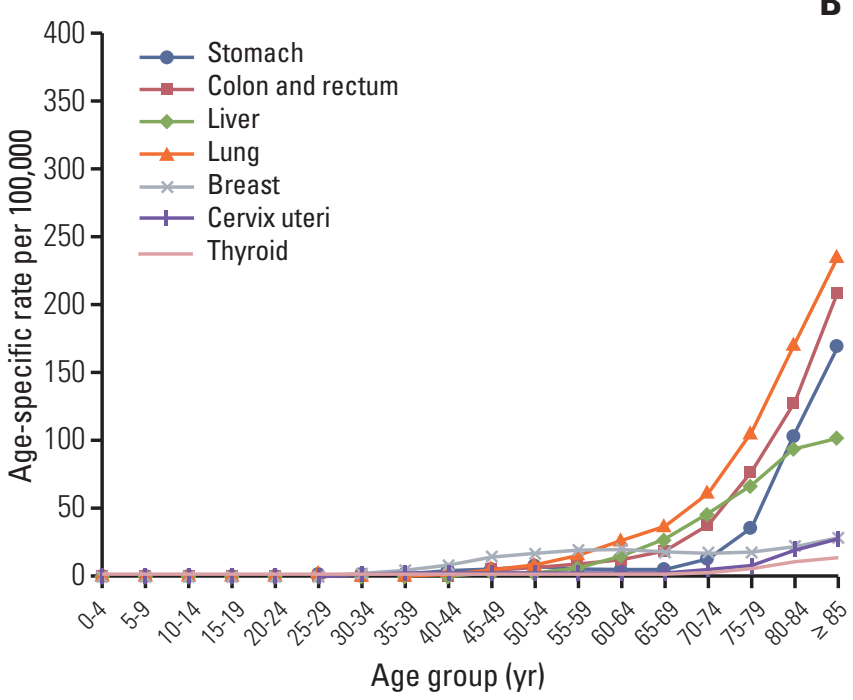

Fig. 3. Projected age-specific mortality rates of major cancers during 2017 in Korea. (A) Male. (B) Female. 
of all sites combined in 2017 for men and women are projected to be 191.5 and 119.2, respectively, whereas the projected ASRs per 100,000 of all sites combined are expected to be 112.7 and 52.2, respectively (Table 4). The five leading cancer sites causing mortality in men are predicted to be lung (CR, 52.5; ASR, 30.1), liver (CR, 31.5; ASR, 18.7), colon and rectum (CR, 21.2; ASR, 12.5), stomach (CR, 18.7; ASR, 11.0), and pancreas (CR, 12.2; ASR, 7.2). During the same period, lung cancer (CR, 19.9; ASR, 8.1) is projected to be the leading cancer cause of death in women, followed by the colon and rectum (CR, 14.5; ASR, 5.7), liver (CR, 11.3; ASR, 4.8), pancreas (CR, 11.2; ASR, 4.6), and stomach (CR, 10.2; ASR, 4.1).

The predicted age-specific mortality rates of the selected cancers in men and women in 2017 are shown in further detail in Fig. 3. When examined by age, lung cancer is expected to have the highest morality rate in Korean men and women aged 60 and over. lung, while lung, colorectal, liver, pancreatic, and stomach cancers are projected to be the most common causes of cancer-related deaths.

Cancer is currently one of the foremost public health concerns in Korea. Although cancer rates are anticipated to decrease somewhat, the burden of most of cancers will continue to grow with aging of the population. The current projections of cancer incidence and mortality for 2017 represent an important resource for planning and evaluation of cancer-control programs. As the estimates in this study are model-based, these results should be interpreted with caution. The incidence of some cancers, such as stomach, colorectum, and thyroid, started to decrease from the early 2010s. As the recent trends of those cancers contained only 4 or 5 data points, therefore their estimates could be unstable.

\section{Conflicts of Interest}

Conflict of interest relevant to this article was not reported.

\section{Acknowledgments} lowed by stomach, colorectal, liver, and prostate cancers. Lung, liver, colorectal, stomach, and pancreatic cancers are expected to be the most common causes of cancer deaths among men. In women, the five leading primary sites are expected to be the breast, thyroid, colorectum, stomach, and
This work was supported by a research grant from the National Cancer Center (No. 1610200), Republic of Korea.

\section{References}

1. Statistics Korea [Internet]. Daejeon: Statistics Korea; 2016 [cited 2017 Feb 15]. Available from: http:// kosis.kr.

2. Oh CM, Won YJ, Jung KW, Kong HJ, Cho H, Lee JK, et al. Cancer statistics in Korea: incidence, mortality, survival, and prevalence in 2013. Cancer Res Treat. 2016;48:436-50.

3. Shin HR, Won YJ, Jung KW, Kong HJ, Yim SH, Lee JK, et al. Nationwide cancer incidence in Korea, 1999 2001: first result using the national cancer incidence database. Cancer Res Treat. 2005;37:325-31.

4. Fritz A, Percy C, Jack A, Shanmugaratnam K, Sobin L, Parkin $\mathrm{DM}$, et al. International classification of diseases for oncology. 3rd ed. Geneva: World Health Organization; 2000.

5. World Health Organization. International statistical classifica- tion of diseases and related health problems. 10th rev. Geneva: World Health Organization; 1994.

6. Boyle P, Parkin DM. Statistical methods for registries. In: Jensen OM, Parkin DM, MacLennan R, Muir CS, Skeet RG, editors. Cancer registration: principles and methods. IARC Scientific Publication. No. 95. Lyon: IARC; 1991. p. 126-58.

7. National Cancer Institute. Joinpoint regression program, version 4.0 [Internet]. Bethesda, MD: National Cancer Institute; c2015 [cited 2015 Jan 8]. Available from: http://surveillance. cancer.gov/joinpoint/.

8. Segi M. Cancer mortality for selected sites in 24 countries (1950-1957). Sendai: Tohoku University School of Medicine; 1960. 